\title{
A continuum model in traffic flow considering the jerk effect
}

\author{
Liu Huaqing \& Ye Caihong \& Ge Hongxia, Yu Chenyan
}

Faculty of Maritime and Transportation, Ningbo University, Ningbo 315211, China

Jiangsu Key laboratory of Urban ITS, Southeast University, Nanjing 210096, China

National Traffic Management Engineering and Technology Research Center, Ningbo University

Sub-center, Ningbo 315211, China

Department of Fundamental course, Ningbo Institute of Technology, Zhengjiang University, Ningbo 315100, China

Keywords: Traffic flow; Continuum model; Traffic Jerk; KdV-Burgers equation

\begin{abstract}
Based on the optimal velocity model, a new continuum model considering the jerk term is mentioned in this paper. Then, the critical condition for the steady traffic flow is deduced. Near the neutral stability line, nonlinear analysis is taken to derive the $\mathrm{KdV}$-Burgers equation for describing the traffic density and one of the solutions is given.
\end{abstract}

\section{The continuum model}

Based on the OVM model[1], the jerk term is added to the dynamic equation, which is

$$
\left\{\begin{array}{c}
\frac{d v_{n}(t)}{d t}=a\left[V\left(\Delta x_{n}(t)\right)-v_{n}(t)\right]-\lambda J_{n}(t) \\
J_{n}(t)=\frac{d v_{n}(t)}{d t}-\frac{d v_{n}(t-1)}{d t}
\end{array}\right.
$$

in which $V$ means the optimal velocity given by Bando and the $\lambda$ is the jerk parameter.

Considering the headway - density formula given by Berg et al. [2]

$$
\Delta x \approx \frac{1}{\rho}-\frac{\rho_{x}}{2 \rho^{3}}-\frac{\rho_{x x}}{6 \rho^{4}}
$$

and the relationship transferring microscopic variables in Eq.(1) into the macroscopic ones as below $v_{n}(t) \rightarrow v(x, t), v_{n+1}(t) \rightarrow v(x+\Delta, t)$,

$$
V\left(\frac{1}{\rho}\right) \rightarrow V_{e}(\rho), V^{\prime}\left(\frac{1}{\rho}\right) \rightarrow-\rho^{2} V_{e}^{\prime}(\rho)
$$

and the Taylor expansions, Eq.(1) is rewritten as follows

$$
\frac{\partial v}{\partial t}+v \frac{\partial v}{\partial x}=a\left[V_{e}(\rho)-v\right]-\lambda v v_{x t}+a V_{e}^{\prime}(\rho)\left(\frac{\rho_{x}}{2 \rho}+\frac{\rho_{x x}}{6 \rho^{2}}\right)
$$

Including the following continuity equation,

$$
\frac{\partial v}{\partial t}+\frac{\partial(\rho v)}{\partial x}=0
$$

We obtain the new model described by Eqs. (4)-(5).

\section{Stability analysis}

For the convenience of analysis, we rewrite the Eqs. (4) and (5) as follow:

$$
\frac{\partial \vec{U}}{\partial t}+\vec{A} \frac{\partial \vec{U}}{\partial x}=\vec{E}
$$

The eigenvalues of the matrix $\vec{A}$ are obtained as follows 
$\lambda_{1}=\lambda_{2}=v$

From Eq.(7), we can see that the characteristic speeds $d x / d t$ are equal to the macroscopic flow speed $v$, which demonstrates the fundamental principle that vehicle flows are anisotropic and response only to frontal stimuli.

Then, we use linear stability method to analyze the qualitative properties of this new model. Considering that the system is a uniform flow, and we apply a infinitesimally perturbation to the homogenous flow

$\left(\begin{array}{l}\rho(\mathrm{x}, \mathrm{t}) \\ v(\mathrm{x}, \mathrm{t})\end{array}\right)=\left(\begin{array}{l}\rho_{0} \\ v_{0}\end{array}\right)+\sum\left(\begin{array}{c}\hat{\rho_{\mathrm{k}}} \\ \hat{v_{\mathrm{k}}}\end{array}\right) \exp \left(\mathrm{ikx}+\sigma_{\mathrm{k}} \mathrm{t}\right)$

With the replacement of $\rho$ and $v$, the Eqs.(6) and (5) will be rewrite without the nonlinear higher-order terms as follows

$$
\left\{\begin{array}{c}
\left(\sigma_{\mathrm{k}}+\mathrm{ik} v_{0}\right) \hat{\rho}_{\mathrm{k}}+\mathrm{ik} \rho_{0} \hat{v_{\mathrm{k}}}=0 \\
\sigma_{\mathrm{k}} \hat{v_{\mathrm{k}}}+v_{0} \hat{v_{\mathrm{k}}} \mathrm{ik}=\mathrm{a}\left[\hat{\rho}_{k} \mathrm{~V}_{\mathrm{e}}^{\prime}\left(\rho_{0}\right)-\hat{v_{\mathrm{k}}}\right]-\lambda v_{0} \hat{v}_{\mathrm{k}} \mathrm{ik} \sigma_{k}(9) \\
+a \hat{\rho_{k}}\left(\frac{V_{e}^{\prime}\left(\rho_{0}\right)}{2 \rho_{0}} i k+\frac{V_{e}^{\prime}\left(\rho_{0}\right)}{6 \rho_{0}^{2}}(i k)^{2}\right)
\end{array}\right.
$$

Taking $\hat{\rho}_{\mathrm{k}}$ and $\hat{v_{\mathrm{k}}}$ as the unknown quantities of the equations, we can obtain that the unknown quantity $\sigma_{\mathrm{k}}$ satisfies the following quadratic equation

$\left(\sigma_{\mathrm{k}}+i k v_{0}\right)^{2}+\left(a+\lambda v_{0} i k \sigma_{k}\right)\left(\sigma_{\mathrm{k}}+i k v_{0}\right)+$

$i k \rho_{0} a V_{e}^{\prime}\left(\rho_{0}\right)\left[1+\frac{i k}{2 \rho_{0}}+\frac{(i k)^{2}}{6 \rho_{0}^{2}}\right]=0$

In the state that both roots of $\sigma_{\mathrm{k}}$ have negative real parts the stable traffic flow will be obtained.

$a_{s}=-2 \rho_{0}^{2} V_{e}^{\prime}\left(\rho_{0}\right)\left[1-\frac{k^{2}}{6 \rho_{0}^{2}}\right]^{2}$

And

$\operatorname{Im}\left(\sigma_{k}\right)=-k\left[v_{0}+\rho_{0} V_{e}^{\prime}\left(\rho_{0}\right)\right]+o\left(k^{3}\right)$

Considering Eq.(12), the critical speed of disturbance propagation will be obtained as $c\left(\rho_{0}\right)=v_{0}+\rho_{0} V_{e}^{\prime}\left(\rho_{0}\right)$

which is mentioned in Ref.[3].

\section{Nonlinear analysis}

The system behavior around the neutral stability condition attracts our attention. Then the role of time $t$ and space $x$ is concentrated in the variant $z$ [4]

$\mathrm{z}=\mathrm{x}-\mathrm{ct}$

So we can rewrite the Eqs. (4) and (5)

- $\mathrm{c} \rho_{\mathrm{z}}+\mathrm{q}_{\mathrm{z}}=0$

$-\mathrm{c} v_{\mathrm{z}}+v v_{\mathrm{z}}=a\left[V_{e}(\rho)-v\right]-\lambda v\left(-c v_{z z}\right)$

$+a V_{e}^{\prime}(\rho)\left(\frac{\rho_{x}}{2 \rho}+\frac{\rho_{x x}}{6 \rho^{2}}\right)$

where $q$ is the product of density and velocity. Also, we can get

$v_{\mathrm{z}}=\frac{c \rho_{\mathrm{z}}}{\rho}-\frac{q \rho_{\mathrm{z}}}{\rho^{2}}$ 
We expand the flow $q$ as

$\mathrm{q}=\rho V_{e}(\rho)+b_{1} \rho_{z}+b_{2} \rho_{z z}$

which represents the flow $q$ with the homogeneous, stability, and stable characters.

Combining the two equations above and introducing them in the Eq.(16), we can get

$-c\left(\frac{c \rho_{z}}{\rho}-\frac{q \rho_{z}}{\rho^{2}}\right)+\frac{q}{\rho}\left(\frac{c \rho_{z}}{\rho}-\frac{q \rho_{z}}{\rho^{2}}\right)=$

$a\left[V_{e}(\rho)-\frac{q}{\rho}\right]-\lambda \frac{q}{\rho}(-c)$

$\left(\frac{c \rho_{z z}}{\rho}-\frac{c \rho_{z}^{2}}{\rho^{2}}-\frac{q \rho_{z z}}{\rho^{2}}-\frac{c \rho_{z}^{2}}{\rho^{2}}+\frac{2 q \rho_{z}^{2}}{\rho^{3}}\right)$

$+a V_{e}^{\prime}(\rho)\left(\frac{\rho_{z}}{2 \rho}+\frac{\rho_{z z}}{6 \rho^{2}}\right)$

Balancing the coefficients of $b_{1}$ and $b_{2}$ in the both sides of Eq.(19), we can deduce

$\left\{\begin{array}{c}b_{1}=\frac{1}{2 a}\left(V_{e}(\rho)-c\right)^{2}+\frac{1}{2} V_{e}^{\prime}(\rho) \\ b_{2}=\frac{V_{e}^{\prime}(\rho)}{6 \rho}+\frac{c \lambda V_{e}(\rho)}{a}\left(c-V_{e}(\rho)\right)\end{array}\right.$

With Taylor expansions, we rewrite Eq.(18) near the neutral stability condition $\rho \mathrm{V}_{e}(\rho) \approx$

$\rho_{h} V_{e}\left(\rho_{h}\right)+\left.\left(\rho V_{e}\right)_{\rho}\right|_{\rho=\rho_{h}} \hat{\rho}+\left.\frac{1}{2}\left(\rho V_{e}\right)_{\rho \rho}\right|_{\rho=\rho_{h}} \hat{\rho}^{2}$

With Eq. (18) and (21), another equation can be deduced

$-c \rho_{\mathrm{z}}+\left[\left(\rho V_{e}\right)_{\rho}+\left(\rho V_{e}\right)_{\rho \rho} \rho \rho_{\mathrm{z}}+b_{1} \rho_{\mathrm{zz}}+b_{2} \rho_{\mathrm{zzz}}\right.$

$=0$

To obtain a regular form, some variable substitutions are taken

$U=-\left\lfloor\left(\rho \mathrm{V}_{\mathrm{e}}\right)_{\rho}+\left(\rho \mathrm{V}_{\mathrm{e}}\right)_{\rho \rho} \rho\right\rfloor \quad X=m s, \quad T=-m t$

Hence, we can deduce the KdV-Burgers equation as follows

$U_{T}+U_{X}-m b_{1} U_{X X}-m^{2} b_{2} U_{X X X}=0$

of which one of the solutions is

$U=-\frac{3\left(-m b_{1}\right)^{2}}{25\left(-m^{2} b_{2}\right)}$

$\left[\begin{array}{l}1+2 \tanh \left( \pm \frac{-m b_{1}}{10 m^{2}}\right)\left(X+\frac{6\left(-m b_{1}\right)^{2}}{25\left(-m^{2} b_{2}\right)^{T}} T+\varsigma_{0}\right)+ \\ \tanh ^{2}\left( \pm \frac{-m b_{1}}{10 m^{2}}\right)\left(X+\frac{6\left(-m b_{1}\right)^{2}}{25\left(-m^{2} b_{2}\right)^{2}} T+\varsigma_{0}\right)\end{array}\right]$

in which $\varsigma_{0}$ is an arbitrary constant.

\section{Summary}

In this paper, the jerk term that indicates the sharp change of accelerated speed in the real traffic has been added to the traditional OVM model. Considering the jerk term, the stability condition of the new OVM model has been studied and then the KdV-Burgers equation has been derived and one of the solutions is given to describe the evolution of density wave happened in the traffic flow.

\section{Acknowledgment}

Project supported by the Science Foundation of Ningbo (Grant Nos. 2012A610162, 
2014A610022 and 2014A6111015), the Scientific Research Fund of Zhejiang Provincial, China (Grant No. LY13A020007), the Open Research Fund of Jiangsu Key laboratory of Urban ITS, Southeast University and the K. C. Wong Magna Fund in Ningbo University, China.

\section{References}

[1] M. Bando, K. Hasebe, A. Nakayama, et al. Dynamical model of traffic congestion and numerical simulation. Phys. Rev. E 1035,51(2) (1995).

[2] P Berg, A. Mason, A. Woods. Continuum approach to car-following models. Phys. Rev. E 1056,61(2) (2000) .

[3] D. Helbing, B. Tilch, Generalized force model of traffic dynamics, Phys. Rev. E 133,58 (1998).

[4] P. Berg, A. Woods, Traveling waves in an optimal velocity model of freeway traffic, Phys. Rev. E 035602,64 (2001).

[5]L.L. Lai, R.J. Cheng, Z.P. Li, The $\mathrm{KdV}$-Burgers equation in a modified speed gradient continuum model. Chinese Physics B 060511,22(6) (2013).

[6]H.X. Ge, L.L. Lai, P.J. Zheng, The KdV-Burgers equation in a new continuum model with consideration of driver's forecast effect and numerical tests. Phys. Letters A 3193-3198,377(44) (2013) . 